\title{
Recognition of "flowering" processes on the base of remote sensing data in shallow water ponds on the example of the Azov Sea
}

\author{
Marina Ganzhur ${ }^{1, *}$, Nikita Dyachenko ${ }^{1}$, Olga Smirnova $^{1}$, Anna Poluyan $^{1}$, Natalya \\ Panasenko ${ }^{1}$ \\ ${ }^{1}$ Don State Technical University, 1, Gagarin sq., 344003, Rostov on Don, Russia
}

\begin{abstract}
This work considers to the processes of «bloom» phytoplankton processes that cause hypoxic phenomena in shallow waters the example of the Sea of Azov. For the accumulation of information, multichannel satellite images of remote sensing are taken as a basis. In the process, the task of programmatically highlighting the contours of the areas of «bloom» is implemented.
\end{abstract}

\section{Introduction}

The World Ocean is a powerful regulator of the balance of oxygen and carbon dioxide in the atmosphere. Its phytoplankton provides $50-70 \%$ of the total amount of oxygen consumed by living beings, the interaction of the ocean and the atmosphere is an important factor for the weather and climate formation [1].

Currently, when environmental problems became of a global level, oceans have taken a leading place in them, since they form the climate and produce a half of the oxygen of our planet. The ocean ecology is directly related to the use of its resources. The importance of the ocean as a huge depot of mineral and biological raw materials is constantly growing, and this gives rise to problems of combating various types of pollution, the need to predict the consequences of scientific and industrial activities of mankind. And that is why there is a need for operational monitoring of the state of our planet.

The model was usually an equation or a system of equations that allowed a solution the dependence of output variables on input ones. Such models are called analytical models. However, not all situations are like that. At the present level of applied research, one often has to deal with complex systems in which there are not only many objective functions, but a kind of obscurity with a quantitative expression of these functions. Here in general, we can not talk about solving various optimization problems as much as about studying complex systems, predicting their future states depending on the chosen control strategies. Since practice urgently required a method for the study of complex systems, it appeared. This method is called simulation modeling.

Let us get on to the description of stages of constructing any mathematical model of the system. It can be imagined as consisting of the following steps:

${ }^{*}$ Corresponding author: mganzhur@yandex.ru 
1. The main questions about the system behavior are formed, the answers to which we want to obtain using the model.

2. Among any laws that manage the system behavior, the ones with essential influence at searching for answers to the questions posed are taken into account.

3. In addition to these laws, if necessary, certain hypotheses about functioning are formulated for the simulated system as a whole or for its individual parts. As a rule, these hypotheses are plausible to the extent that some theoretical arguments can be presented in favor of their adoption.

4. Hypotheses, like laws, are expressed in the form of certain mathematical relationships which are combined into some formal description (formulas, algorithms).

The criterion of adequacy is the practice, which determines when the process of the model improvement can end. There is no need to say that this criterion is not formalized and requires special research in each case.

\section{Accumulation of information using satellites}

The analysis and prediction of natural systems requires the construction of complexes of non-stationary models of hydrophysics, hydrobiology and biogeochemical cycles. Currently, developed models of these processes have been built in Russia, as well as abroad (the USA, Germany, France, and other countries), but the effective use of these models for the purpose of analysis and forecasting faces the problem of providing them with real input data, which allow setting tasks correctly.

To present, the effective use of these models for the purpose of analysis and forecasting is challenged by providing them with real input data. As a source of such input data in modern conditions, it makes sense to use information from Artificial Earth Satellites, as well as from unmanned aerial vehicles and other remote sources of geophysical information [2]. This method has clear advantages: relevance of data at the time of shooting, high efficiency of data collection, economic feasibility etc.

To date, various hardware and technical means are being developed for implementing measures to monitor the sea surface. Data from NOAA, NASA Worlgview, Landsat, SPOT, IRS, RADARSAT, ERS satellites and other data are widespread. The main monitoring data at present are the ones from space-based radars, Synthetic Aperture Radars (SAR) - there are radar images of Radarsat-2, TerraSAR-X, COSMO-SkyMed-1/2/3/4, Sentinel-1A/1B and other satellites. Data from optical sensors, such as MODIS spectroradiometers at Terra, Suomi NPP, Aqua and other satellites, are used when the weather is cloudy. Daily continuous shooting allows accurate determination of the situation in the aquatic environment, as well as the size and configuration of objects (spots of planktonic populations and suspensions, films of oil products, etc.), a change in time and a number of other important features.

\subsection{Description of the implemented software complex}

This study describes the first stage of a complex of intellectual image processing software for objects like plankton populations, distribution of matter suspended in the aquatic environment, distribution of salinity in the near-surface layer. The complex is designed to accumulate data of mathematical modeling of possible scenarios for the development of the ecological situation of coastal systems using the example of the Azov-Black Sea basin. At the development, the high-level language of $\mathrm{CH}$ was used.

For the implementation, we will use an algorithm based on LBP (Local Binary Patterns) attributes. In our case, the LBP attribute is an integer value of 0 or 255 , which reads the 
difference in brightness of the current pixel and other ones in the process of enumerating all the pixels. A detailed description of this algorithm can be found in work [3].

"The LBP-feature (in the OpenCV implementation) is an integer value from to that is calculated based on rectangular image area (which is part of the detection window):

$$
\begin{array}{r}
\operatorname{LBP}(X, Y, W, U)=2^{7} \times\left[S_{00} \geq S_{11}\right]+2^{6} \times\left[S_{10} \geq S_{11}\right]+2^{5} \times\left[S_{20} \geq S_{11}\right]+ \\
2^{0} \times\left[S_{01} \geq S_{11}\right]+2^{4} \times\left[S_{21} \geq S_{11}\right]+2^{1} \times\left[S_{02} \geq S_{11}\right]+2^{2} \times\left[S_{12} \geq S_{11}\right]+2^{3} \times
\end{array}
$$

$$
\left[\mathrm{S}_{22} \geq \mathrm{S}_{11}\right]
$$

Here:

$(\mathrm{X}, \mathrm{Y})$ - coordinates of top-left pixel of rectangular image area for LBP-feature calculation;

$(\mathrm{W}, \mathrm{H})(3 \mathrm{w} .3 \mathrm{~h})$ - size of the area;

$S_{i j}=\sum_{x=0}^{w-1} \sum_{y=0}^{h-1} p(X+i w+x, Y+j h+y)-$ the sum of pixel values over rectangle;

$\mathrm{p}(\mathrm{x}, \mathrm{y})-$ value of pixel in column and row ;

$$
[a \geq b]=\left\{\begin{array}{l}
1, \text { if } a \geq b \\
0, \text { otherwise }
\end{array}\right.
$$

The analysis of multichannel satellite images allows identifying pond areas being the most susceptible to suffocation phenomena. On the pictures, it is possible to track the dynamics of the "flowering water" phenomenon, which caused the fish kill [4].

The results are based on images obtained using the Earth satellite monitoring: SIC "Planet" (a picture dated 05.06.2019 is shown in Figure 1) [5] and NASA Worldview (a picture dated 05.06.2019 is shown in Figure 2) [6].

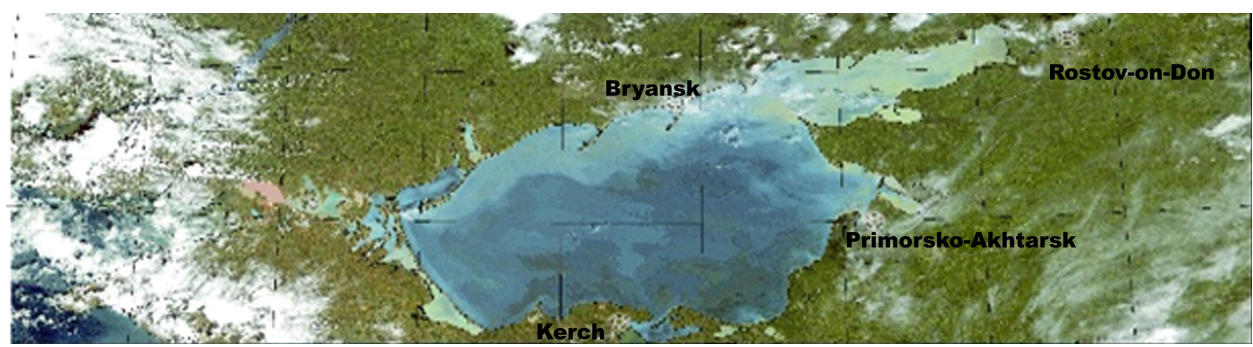

Fig. 1. Picture from SIC "Planet".

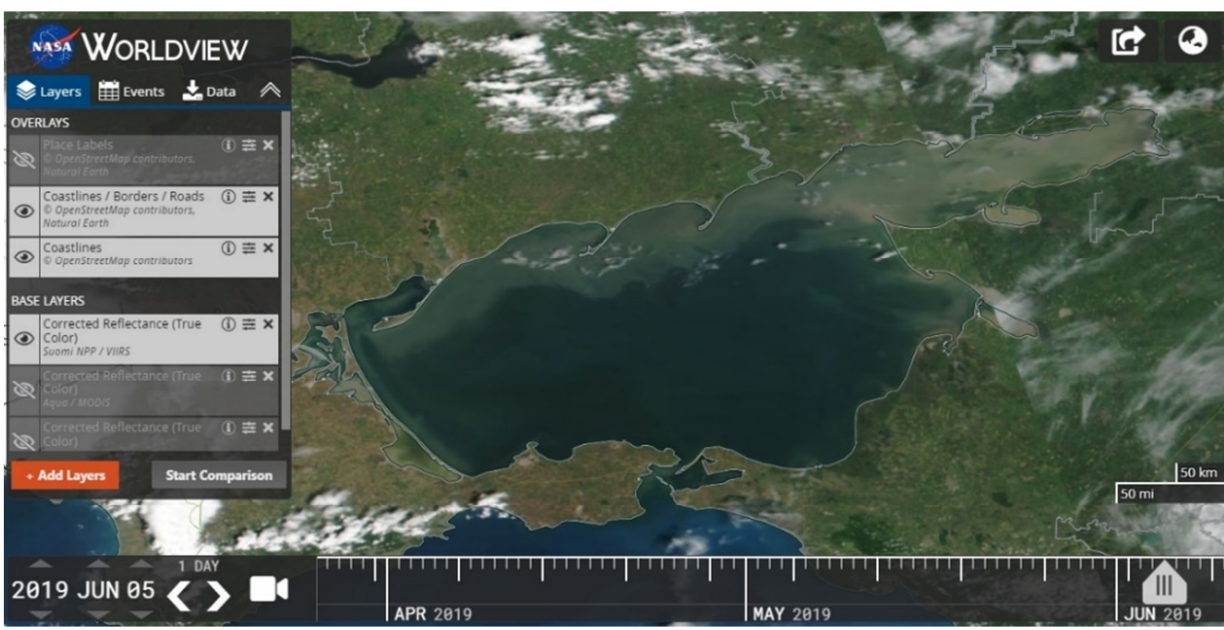

Fig. 2. Picture from NASA Worldview. 
Having selected the area required for work, we will build a grid and highlight the "flowering" area manually for further comparison (Figure 3).

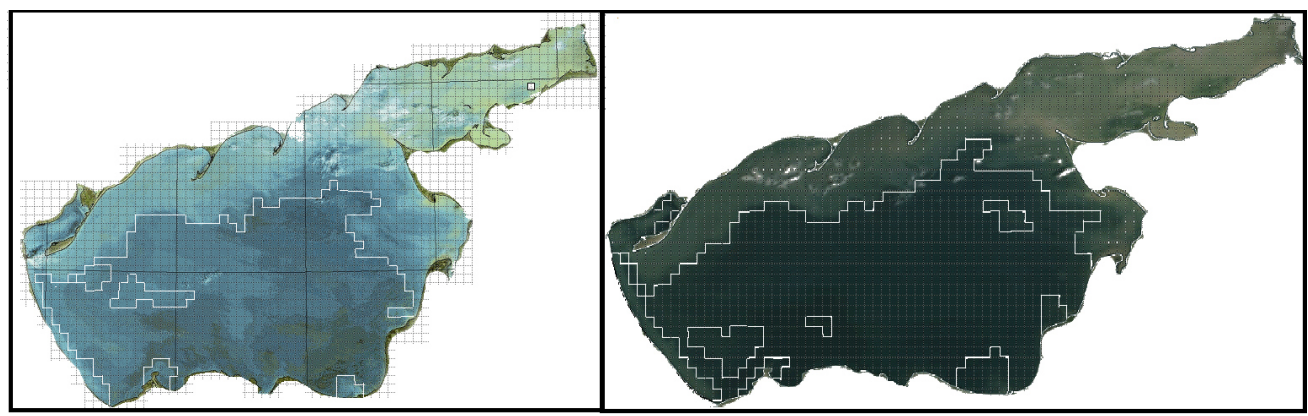

Fig. 3. Manual highlighting of "flowering" areas.

In the process of implementation and setting the required threshold values, the following results will be obtained: Figure 4 without the grid and Figure 5 with the grid.
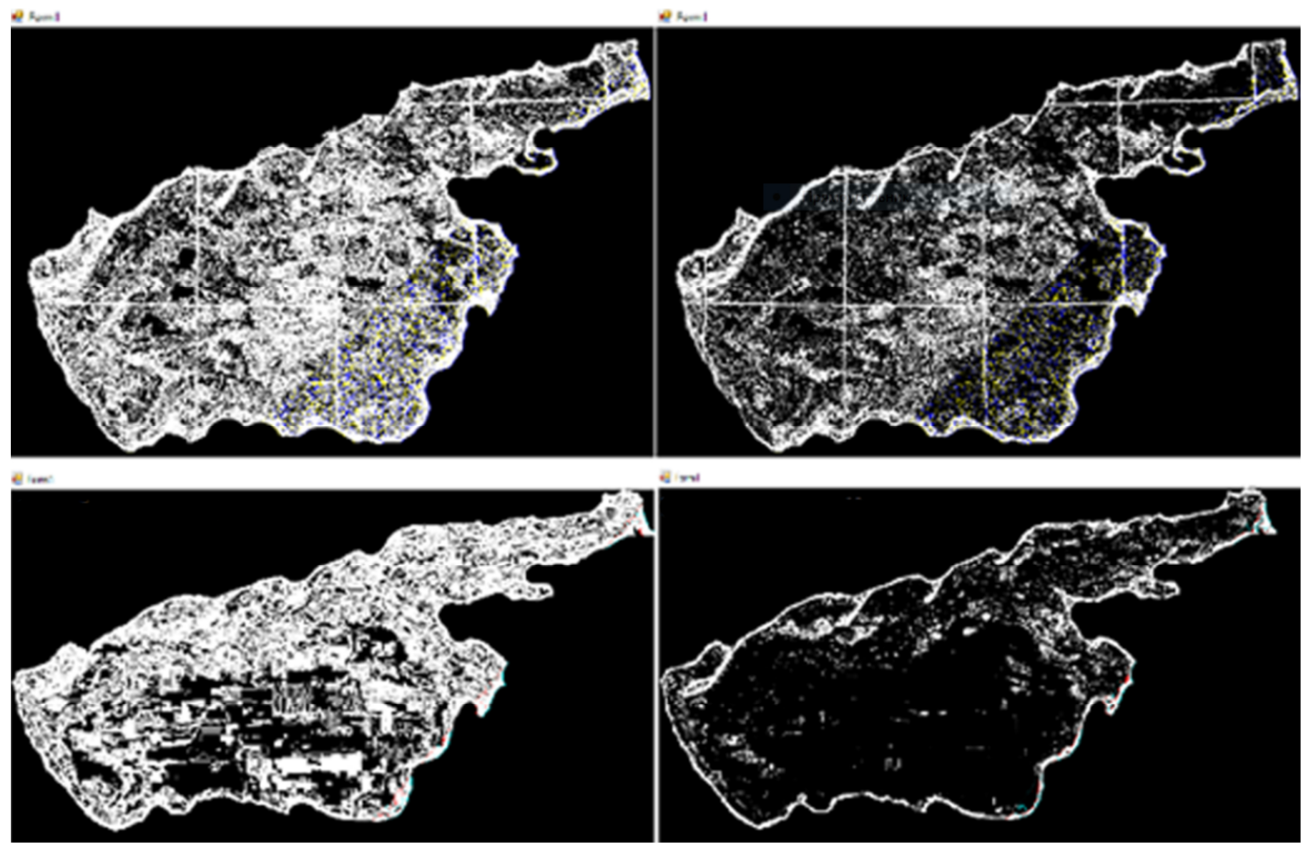

Fig. 4. Highlighting the "flowering" contour using the developed program, without the grid. 

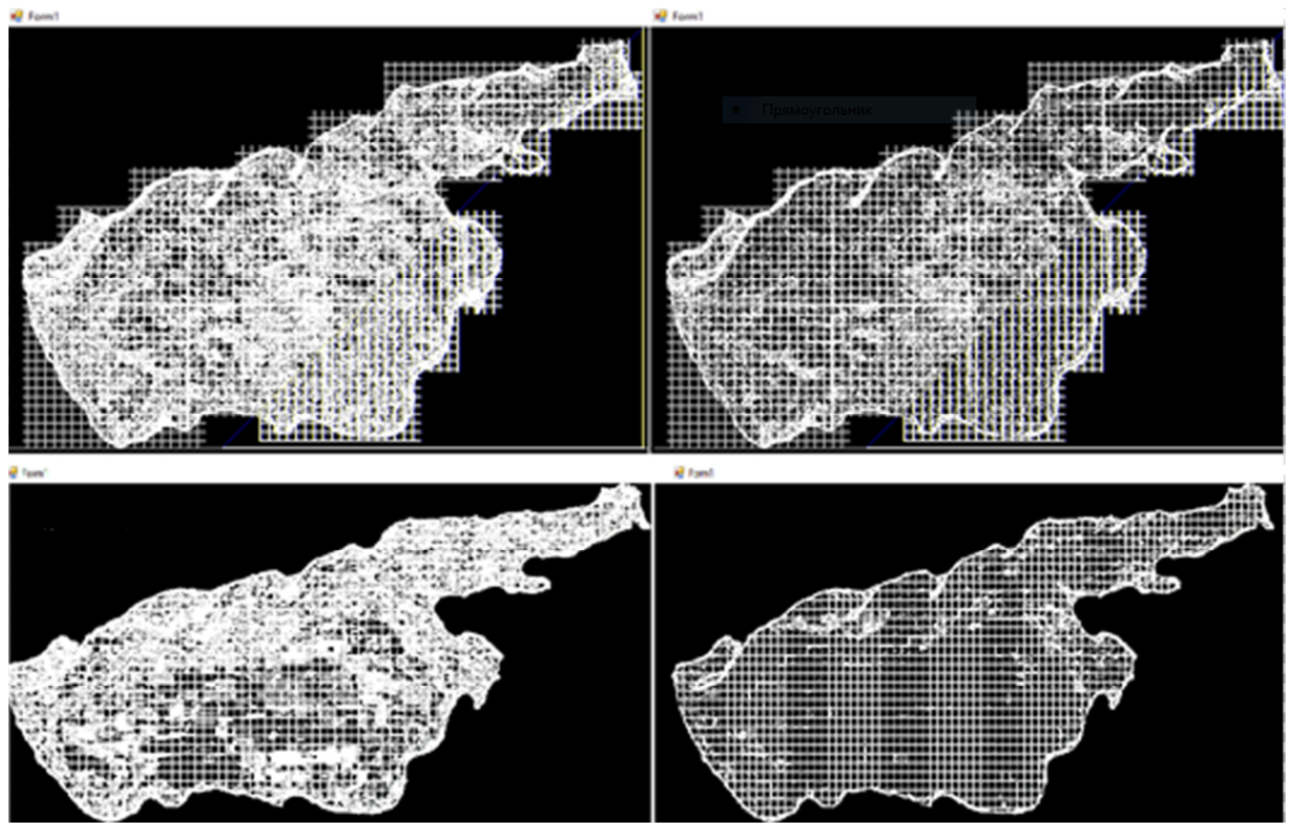

Fig. 5. Highlighting the "flowering" contour using the developed program, with the grid.

The program clearly demonstrates the possibilities of identifying the contours and tracing their change in the course of time on the pond surface. When applying the grid, we clearly see the similarity of the result with the one of manual highlighting. In the future, with the software improvement, it is supposed to finalize the product of mathematical calculations, in particular, the ability to determine the center line of mass of recognized objects.

\section{Conclusion}

The study includes theoretical calculations and justifications for its rationale. The software implementation of recognition of "water flowering" in shallow water ponds is presented. In further studies, it is expected to devote more time to the consideration of the mathematical aspect of this issue and the software complex improvement.

\section{Acknowledgements}

The reported study was funded by RFBR, project number 19-37-90070.

\section{References}

1. N.D. Panasenko, Priority areas for the development of science and technology: dokl. $X X V I$ international scientific and practical. Conf. (Innovative Technologies, Tula, 2019)

2. A.A. Sukhinov, G.B. Ostrobrod, Computational Mathematics and Information Technologies. Electronic journal 1 (2017)

3. A.I. Sukhinov, A.V. Nikitina, A.E. Chistyakov, Computational Mathematics and Information Technologies. Electronic journal 2 (2017) 
4. Federal State Budgetary Institution Research Center Planeta, http://planet.rssi.ru/

5. The official website of NASA Worldview, http://worldview.earthdata.nasa.gov

6. Y.S. Abu-Mostafa, M. Magdon-Ismail, H.-T. Lin, Learning from Data (AMLBook, 2012)

7. A.I. Sukhinov, A.E. Chistyakov, A.A. Semenyakina, A.V. Nikitina, Computer researches and modeling 8(1), 151 - 168 (2016)

8. A.I. Sukhinov, A.V. Nikitina, A.E. Chistyakov, I.S. Semenov, A.A. Semenyakina, D.S. Khachunts, 10th Annual International Scientific Conference on Parallel Computing Technologies, PCT 2016, CEUR Workshop Proceedings 1576, 320-333 (2016)

9. A.V. Nikitina, A.I. Sukhinov, G.A. Ugolnitsky, A.B. Usov, A.E. Chistyakov, M.V. Puchkin, I.S. Semenov, Mathematical Models and Computer Simulations 9(1), 101107 (2017)

10. A.V. Nikitina, M.V. Puchkin, I.S. Semenov, A.I. Sukhinov, G.A. Ugolnitsky, A.B. Usov, A.E. Chistyakov, Management of large systems 55, 343 - 361 (2015)

11. F. Ujoh, I.D. Kwabe, O.O. Ifatimehin, Journal of Geography and Regional Planning 3(5), 106-113 (2010)

12. H.S. Sudhira, S.R. Karthik, K.J. Sanjeev, Urban Sprawl Pattern Analysis using GIS: CES Technical Report 99 (2011)

13. A.I. Sukhinov, A.V. Nikitina, A.E. Chistyakov, I.S. Semenov, Computational methods and programming: new computing technologies 14(1), 103-112 (2013)

14. A.I. Sukhinov, A.E. Chistyakov, E.A. Protsenko, Izvestiya SFU. Technical science 8(121), 159-167 (2011)

15. A.I. Sukhinov, A.E. Chistyakov, E.A. Protsenko, Mathematical models and computer simulations 6(4), 351-363 (2014) 